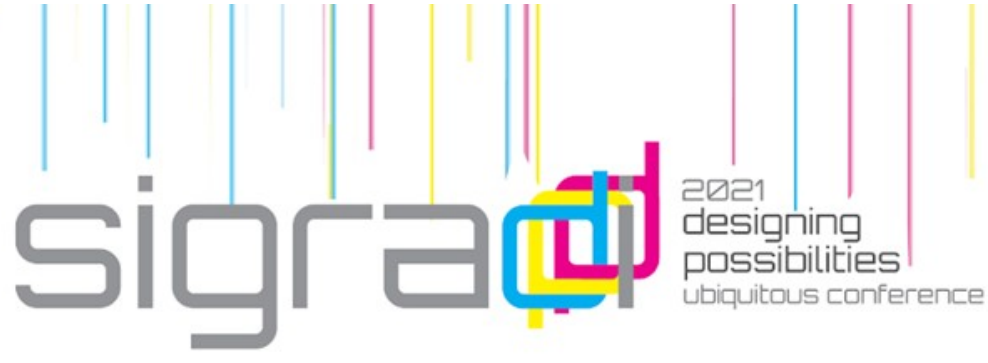

\title{
Pandemic, City and Planning: The Importance of Systematizing Covid-19 Data in Fortaleza
}

\author{
Mateus Soares $^{1}$, Carolina Guimarães ${ }^{1}$, Daniel Cardoso ${ }^{1}$ \\ ${ }^{1}$ Universidade Federal do Ceará, Brasil \\ mateussoares@arquitetura.ufc.br \\ carolina.jorge.guimaraes@gmail.com \\ danielcardoso@ufc.br
}

\begin{abstract}
The COVID-19 pandemic generated great changes in world societies, and Brazilian cities felt strongly the effects of the disease in physical and social aspects. Thus, this article seeks to describe a work experience involving COVID-19 data for use in urban planning analyses. Its main methodology is the treatment of data about the pandemic in the city of Fortaleza and georeferencing these to generate urban readings. The work resulted in a shapefile file that could be shared among those who wanted to research how the dissemination of the new coronavirus was influenced by specific urban issues in the city of Fortaleza, in addition to generating learning for the students involved in the process. From this it was possible to observe the importance of discussions both on urban policies and the importance of data in urban planning models adopted in the country in view of the new pandemic situation.
\end{abstract}

Keywords: Pandemic, COVID-19, Urban planning, Information modeling, Data base

\section{Introdução}

Em 2020, a pandemia da Covid-19 surpreendeu o mundo e exacerbou uma série de problemáticas sistêmicas. $\mathrm{O}$ acontecimento colocou o funcionamento das estruturas sociais, econômicas e institucionais, tanto a nível local como global, sob circunstâncias desafiadoras, o que permitiu importantes reflexões sobre as lógicas as quais a sociedade opera e se organiza.

Ainda em março de 2020, David Harvey fez uma leitura da pandemia focando no seu impacto nos sistemas financeiros globais e na facilidade de espraiamento do vírus, considerando o mundo extremamente conectado da 
atualidade, o despreparo para o enfrentamento de crises sanitárias que anos de políticas de austeridade na saúde pública provocaram e como a doença é sofrida de formas distintas a partir das diferenças de classe. O teórico britânico aponta para uma correlação entre as implicações do vírus e as vulnerabilidades presentes no modelo econômico hegemônico (Harvey, 2020).

Com o avanço do vírus no Brasil foi possível traçar relações entre a disseminação e os impactos da pandemia e as desigualdades sociais do país. A presença de fatores de risco da doença - idade acima de 60 anos e presença de comorbidades -, por exemplo, são maiores na população de baixa renda (Carvalho, 2020). Além disso, no que tange às nossas cidades, especialmente os grandes centros urbanos, a presença de assentamentos precários e a insegurança sanitária de suas habitações são fatores que agravaram a crise em determinados territórios (Observatório das Metrópoles, 2020; Tonucci, 2020).

A pandemia evidenciou as disjunções estruturais do espaço urbano brasileiro, as quais se revelam nas problemáticas socioeconômicas e se relacionam diretamente com a capacidade de determinadas populações enfrentarem crises. São essas reflexões que orientam para a necessidade de espacializar os dados da Covid-19, permitindo leituras territoriais.

Frente ao cenário apresentado, é evidente que a pandemia da Covid-19 torna-se uma matéria a ser tratada pelo urbanismo. O planejamento urbano tem como tarefa orientar o desenvolvimento das cidades objetivando a melhoria da qualidade de vida de todos os seus moradores. A leitura espacial das consequências da atual crise sanitária pode alimentar e reforçar as tomadas de decisão.

De acordo com Ascher, o novo urbanismo deve estar preparado para atuar em atitudes reflexivas e adaptadas a uma sociedade complexa e um futuro incerto, de forma a gerar múltiplos cenários e trabalhar em cadeia cíclica (feedback da cibernética) no planejamento urbano para que esse consiga atender à complexidade da sociedade contemporânea (Ascher, 2010). A partir da compreensão do momento emergencial em questão, essa abordagem tem potencial para responder às futuras crises e às rápidas transformações às quais as nossas cidades estão sujeitas.

A vida na cidade é dinâmica e a prática do planejamento deve estar alinhada a esse aspecto. Assim, a tecnologia da informação e o Big Data mostram-se como ferramentas cada vez mais relevantes para planejar cidades mais resilientes e habitáveis (ETH, n.d.). Isso posiciona os estudos sobre produção e manipulação de dados dentro da seara do planejamento urbano. 
A partir da importância da modelagem da informação diante do que foi apresentado, o presente trabalho tem como objetivo descrever o processo de tratamento dos dados da Covid-19 na cidade de Fortaleza, Ceará, pelo ArqPET $^{1}$ da Universidade Federal do Ceará (UFC). Inicialmente serão

apresentados os objetivos gerais e específicos, para então relatar a metodologia utilizada e, por fim, expor os resultados e as reflexões geradas.

\section{Objetivos}

\subsection{Geral}

Podemos destacar como principal objetivo do presente artigo a descrição do processo de tratamento dos dados referentes a COVID-19 na cidade de Fortaleza, Ceará, Brasil. O trabalho produziu um arquivo georreferenciado que contém a espacialização dos casos de COVID-19 da cidade de acordo com as divisões de cada bairro. O intuito era desenvolver dados que pudessem ser lidos de forma mais visual, gerando então um arquivo shapefile a partir de uma planilha tratada e montada pela equipe do ArqPET UFC ${ }^{1}$ com base nos dados divulgados através da plataforma Integrasus ${ }^{2}$.

É válido ressaltar que para a viabilização do processo foi de extrema importância a disponibilização dos dados da pandemia de forma aberta e organizada. Diante disso, o estado do Ceará apresentou um dos melhores índices de transparência da COVID-19 segundo estudo desenvolvido pela Open Knowledge Brasil (OKBR) para avaliar a qualidade dos dados e informações da pandemia do novo Coronavírus publicados pela União e pelos estados brasileiros nos portais oficiais.

Apesar da transparência, essas informações estavam fragmentadas e pouco compreensíveis no momento em que o trabalho foi desenvolvido. Para sua apreensão e maior alcance houve a necessidade de sistematizar os dados através de representações gráficas-visuais, entendendo como se deu a evolução dos casos diante do tecido urbano extremamente desigual,

1 O Programa de Educação Tutorial do curso de Arquitetura e Urbanismo da Universidade Federal do Ceará é um programa extracurricular que atua nos eixos da pesquisa, do ensino e da extensão. Composto por alunos da graduação e um professor tutor, o grupo realiza atividades focadas na área do urbanismo, com foco em comunidades e assentamentos informais.

Portal: http://pet.arquitetura.ufc.br/p/sobre-o-arqpet.html

${ }^{2} \mathrm{O}$ IntegraSUS é uma plataforma de transparência da gestão pública de saúde do Estado do Ceará.

Acesse em: https://integrasus.saude.ce.gov.br/\#/home 
levando em consideração fatores espaciais e estatísticas urbanísticas que as cidades brasileiras apresentam. Isso tudo motivou o grupo a desenvolver a pesquisa com os dados da Covid-19 na cidade de Fortaleza.

\subsection{Específicos}

Os objetivos específicos buscam detalhar algumas das intenções existentes quando o presente trabalho foi elaborado. A primeira delas era permitir de forma ampla análises socioespaciais no recorte da cidade de Fortaleza a partir da nova e alarmante condição pandêmica, sendo submetido com o intuito de gerar visualização geoespacial de como o COVID-19 se comportaria em um espaço urbano pautado na desigualdade social.

A espacialização dos dados referentes à doença poderia ser sobreposta a outros dados como os de acesso a infraestruturas, renda, regularidade fundiária, dentre outras informações que viessem a ser pertinentes. O objetivo era traçar um paralelo entre espaços com padrões urbanísticos inferiores aos mínimos exigidos pelas legislações e a disseminação do vírus do COVID-19 na cidade.

Era também objetivo do grupo ampliar o debate sobre a importância dos dados nos novos modelos de planejamento urbano pautados no feedback da cibernética, de forma a gerar cenários e melhor compreender uma sociedade que se comporta de maneira complexa e produz futuros incertos. O novo urbanismo deve adaptar-se à nova realidade e trazer novos modelos de análise e de planejamento (Ascher, 2010). Assim, a utilização dos dados tratados e espacializados pode somar ao presente discurso, ao passo que a pandemia traz novos parâmetros urgências e desafios.

À vista disso, é levantada a importância da obtenção de dados de forma aberta e com o acesso facilitado. Assim, outra intenção do grupo é disponibilizar os dados e informações para aqueles que desejam pesquisar e se utilizar dos mesmos para qualquer outro objetivo. Por último, a equipe de trabalho buscou, através da experiência e do aprendizado coletivo, absorver os conhecimentos aqui disseminados, tanto acerca da importância de dados e cenários no planejamento urbano como o tratamento de dados abertos e transformação desses em material para análise urbana.

\section{Metodologia}

Em março de 2020, quando o Covid-19 chegou ao Brasil, as discussões sobre os impactos do coronavírus nas cidades brasileiras se intensificaram e 
as consequências em Fortaleza começaram a ser sentidas. É nesse cenário que o ArqPET UFC decidiu alinhar suas atividades à atual crise e criou a plataforma $\mathrm{SOMAR}^{3}$. Dentre as ações, o grupo de trabalho sentiu a necessidade de produzir leituras espaciais da doença, o que levou aos objetivos mencionados anteriormente.

Para análise e tratamento dos dados foi estabelecido o recorte temporal de março a junho de 2020, o qual configura o momento de início e pico da primeira onda na capital. Nesse período, o poder público emitiu boletins epidemiológicos ${ }^{4}$ semanais, cujos quais já apresentavam uma espacialização dos casos e óbitos no território da cidade. No entanto, esses dados não eram disponibilizados em um formato manipulável que permitisse sua aplicação em softwares de geoprocessamento e análises independentes da equipe.

A atividade, então, foi dividida em duas principais etapas, a de coleta e tratamento dos dados e a de geoprocessamento e produção do shape final, para posteriormente permitir as análises espaciais. $O$ processo de coleta se deu através da plataforma do IntegrasuS, o site deu acesso às planilhas que continham os dados de casos e óbitos por indivíduo em todo o estado do Ceará, com as variáveis de município, bairro, sexo, idade do paciente, data do início dos sintomas, data da notificação, resultado final do exame e evolução do caso. Esse documento era atualizado de acordo com a publicação dos boletins epidemiológicos. A planilha utilizada neste trabalho compila os casos até o dia primeiro de julho.

A opção de acessar os dados da fonte original, invés de coletá-los já tratados e de forma manual a partir dos boletins, deu-se com o intuito de iniciar o trabalho "do zero", sem a sobreposição de processos já realizados por outros agentes. Esse aprofundamento na produção dos dados da saúde exerceu tanto um papel de exercício educativo, quanto uma tentativa de romper com as "caixas pretas" de modelos de produção da informação (Crawford, 2016 apud Furtado e Renski, 2019).

A princípio, a planilha do IntegraSUS parecia permitir uma série de análises visto o nível de desagregação da informação, por indivíduo, e as variáveis existentes. Isso retrata o bom nível de transparência dos dados da pandemia no estado do Ceará, como já apresentado anteriormente, e reforça a importância de um sistema de dados integrado e em rede para produzir estatísticas cada vez mais coerentes com a realidade, principalmente em contextos emergenciais. No entanto, ainda na fase inicial, a equipe de

\footnotetext{
3 “... a plataforma SOMAR busca unir e mapear informações, divulgar ações solidárias e refletir sobre a situação das comunidades em Fortaleza durante a crise do Coronavírus." Acesse em: https://www.somarqpet.orql

${ }^{4}$ Acesse em: https://coronavirus.fortaleza.ce.gov.br/boletim-epidemiologico.html
} 
trabalho pôde verificar a falta de rigor para o preenchimento dos dados na tabela, encontrando muitas células em branco, preenchidas de forma errada ou sem padrão, fato que acaba por prejudicar a precisão das informações geradas no final.

Dessa forma, para a primeira etapa da atividade foi primordial a limpeza e padronização da planilha, na qual foi feita a filtragem dos casos localizados no município de Fortaleza e a padronização do nome dos bairros, escritos de diversas formas diferentes na tabela original. Durante o processo de normatização do nome dos bairros, foi definido que as células preenchidas com nomes de comunidades (assentamentos precários) que poderiam ser encontrados em arquivos oficiais seriam substituídas pelo bairro correspondentes, bem como células vazias ou preenchidas com nome de logradouros, números ou lugares não identificados seriam classificadas como "não identificadas". Além disso, foi possível verificar a existência de pacientes cujos casos, mesmo contendo a data de notificação dos exames, não apresentaram informações sobre o resultado do exame (positivo, negativo ou em análise), o que pode indicar tanto a defasagem no preenchimento da planilha quanto a falta de testes.

O trabalho de limpeza e padronização da tabela certamente foi a fase mais demorada por exigir um esforço operacional maior e menos automatizado, além de requerer tomadas de decisão mais pragmáticas em relação ao que seria contabilizado ou não. Dentre as 138.587 células dentro do município de Fortaleza na tabela , 34.688 não têm identificação de lugar e 42.301 não têm informação sobre o resultado final do exame, cerca de $25 \%$ e $30 \%$ das células respectivamente. São vazios que configuram uma margem de erro, tanto para o estudo em questão quanto para os resultados dos boletins epidemiológicos.

Concluída essa parte, ainda na etapa de coleta e tratamento dos dados, foi iniciada a produção de uma nova tabela que fizesse a somatória dos casos e óbitos por bairro separados por mês, de março a junho. Quantificamos os casos a partir das colunas que informavam a data do início dos sintomas e o resultado final do exame, sendo o último preenchido com "positivo", e os óbitos partindo das colunas de data do óbito e evolução do caso, sendo o último preenchido com "óbito". Essa metodologia de contagem é semelhante ao que está indicado no Dicionário de Dados do IntegraSuS ${ }^{5}$, com uma diferença na contabilização dos óbitos diante da necessidade de separarmos essa informação por data.

\footnotetext{
${ }^{5}$ Acesse em:

https://integrasus.saude.ce.qov.br/\#/indicadores/indicadores-coronavirus/indice-transp arencia. Atualmente, algumas metodologias foram modificadas e ajustadas.
} 
A primeira etapa do trabalho foi superada mediante a finalização da tabela, todas as operações foram realizadas no Excel e Google Sheets de forma coletiva por cerca de 10 alunos, visto a demanda de padronização manual. Essa fase foi permeada por inquietações do grupo acerca do processo de coleta e tratamento de dados, provocando uma compreensão maior da complexidade desse modelo de produção de informação. A tabela final se estrutura como mostra a Figura 01.

A segunda etapa foi mais rápida e simples de ser executada, considerando os conhecimentos do software Qgis e da extensão Postgresql da equipe. Assim, para tornar a tabela de casos e óbitos visível espacialmente no Qgis, é necessário uma geometria com coordenadas específicas para cada bairro associada à ela. Com essa finalidade, foi utilizado o arquivo em shape dos bairros oficiais da cidade, disponibilizado pelo Fortaleza em Mapas, para encontrar as coordenadas geográficas dos centróides e geometria da poligonal de cada bairro.

Tabela 1. Tabela produzida pela equipe do ArqPET com os dados da COVID-19 da cidade de Fortaleza. ArqPET UFC, 2020.

\begin{tabular}{|l|l|}
\hline \multicolumn{1}{|c|}{ Coluna } & Id \\
\hline id & nome do bairro \\
\hline bairro & número de casos acumulados até dia 01/04/2020 \\
\hline casosmarco & número de óbitos acumulados até dia 01/04/2020 \\
\hline obitosmarco & número de curas acumuladas até dia 01/04/2020 \\
\hline curasmarco & número de casos acumulados até dia 01/05/2020 \\
\hline casosabril & número de óbitos acumulados até dia 01/05/2020 \\
\hline obitosabril & número de curas acumuladas até dia 01/05/2020 \\
\hline curasabril & número de casos acumulados até dia 01/06/2020 \\
\hline casosmaio & número de óbitos acumulados até dia 01/06/2020 \\
\hline obitosmaio & número de curas acumuladas até dia 01/06/2020 \\
\hline curasmaio & número de casos acumulados até dia 01/07/2020 \\
\hline casosjunho & número de óbitos acumulados até dia 01/07/2020 \\
\hline obitosjunho & número de curas acumuladas até dia 01/07/2020 \\
\hline curasjunho & número de casos acumulados até dia 10/07/2020 e sem data atribuída, total de casos \\
\hline registrados na planilha do IntegraSUS
\end{tabular}

Posteriormente, esse shapefile foi transferido para o banco de dados da UFC através do Postgis, um plugin que conecta o Qgis ao Postgresql. A primeira tabela criada pelo grupo com os dados relacionados ao COVID-19 (Tabela 1) também foi transferida. Tendo essas duas tabelas inseridas no banco de dados e com as colunas de "bairro" correlacionadas, foi possível, a partir de códigos de SQL, concatenar as duas informações gerando uma 
nova planilha na qual as informações dos casos, óbitos e curas estivessem associadas às geometrias, aos nomes e aos ids de cada bairro de Fortaleza.

Importante destacar que as colunas de "id" e "bairro" estavam correlacionadas e as duas existiam tanto na planilha com a informação das geometrias dos bairros como na planilha com as informações sobre a pandemia de COVID-19 (Tabela 1). A partir dessas colunas que foi possível ser feito a concatenação das informações, havendo relação entre a informação de número, presente na coluna "id", com a informação de texto (name) presente na coluna de "bairro".

Por fim, vale ressaltar que as informações produzidas foram comparadas aos resultados dos boletins epidemiológicos emitidos pela prefeitura, sendo encontradas pequenas diferenças entre quantidade de dados presentes em cada uma das fontes de informação. No entanto, a diferença era desconsiderável e muito provavelmente gerada pela quantidade de erros de preenchimento dos dados encontrados na base original do Integrasus e pela minúcia no tratamento desses dados que o grupo teve. Assim, os dados tratados e organizados pela equipe do ArqPET mostraram-se bem próximos daqueles que eram informados pelos canais oficiais gerando validação para a informação produzida na academia.

\section{$4 \quad$ Resultados e Discussões}

O trabalho conseguiu atingir alguns resultados pertinentes sendo o primeiro deles a construção e finalização do arquivo shapefile contendo as informações relacionados ao COVID-19 como descrito e apresentado no tópico de metodologia. Para além do shape foi elaborado um relatório da metodologia de tratamento dos dados e outro relatório com as informações referentes às colunas de linhas existentes na planilha final que deu origem ao shape. O shape foi disponibilizado para aquele que tivesse interesse em pesquisar junto dos relatórios para auxiliar no entendimento do mesmo.

Dessa maneira, algumas ações foram tomadas após a finalização da planilha, foi realizado também dentro da plataforma SOMAR que iria produzir uma série histórica com o acumulado de casos, novos casos, óbitos e curas por COVID-19 (Figuras 1 e 2) entrelaçados a outros dados como o de assentamentos precários na cidade de Fortaleza. A ação tem o objetivo de entender e verificar as fragilidades geradas pelo planejamento dito funcionalista que oculta a cidade periférica e a deixa escassa de infraestrutura, resultando em um vulnerabilidade ainda maior para esses espaços nesse momento de pandemia. 
Isso se deve pelo histórico processo de planejamento feito no brasil, que é aquele que mostra a existência de um pântano entre sua retórica e sua prática, imerso em uma base fundante marcada por contradições: direitos universais, normatividade cidadã - no texto e no discurso - versus cooptação, favor, discriminação e desigualdade - na prática da gestão urbana (Maricato, 2002). Considerando a discussão sobre esse modelo de planejamento urbano e as problemáticas do mesmo, que só ficou ainda mais explícito diante do contexto pandêmico, tornou-se evidente a importância da garantia de uma cidade justa e inclusiva mediante a produção de dados que possam gerar uma imagem que embase todas essas questões.

Ademais os dados publicados nos boletins epidemiológicos não eram em sua grande maioria dados extremamente organizados, existiam alguns ruídos que eram gerados no momento de preenchimento das tabelas em hospitais ou locais de atendimento a pacientes com COVID-19, prejudicando a rede de dados e gerando alguns áreas cinzas nos resultados finais de análise. Tal fato reforça a relevância de uma rede de dados em que todos os envolvidos estejam capacitados para a tarefa, sem comprometer o potencial da informação.

Há também a problemática em relação ao domínio da informação. Dados são produzidos e manipulados por grupos que detêm o poder, havendo sempre de ter o cuidado em gerar análises a partir de dados que não perpetuem políticas que excluem certas populações dos espaços de poder e as invisibiliza para fazer reivindicações (Rosenström, 2006). Um fato que pode auxiliar nessa reflexão é o de que o grupo encontrou um número de casos maior que o apresentado nos boletins epidemiológicos, isso pode ter se dado pelo fato de, durante o trabalho manual de padronização, ter sido considerado os indivíduos que deram o nome de suas comunidades, pacientes que podem não ter entrado no processo automatizado de contabilização do poder público.

Outro questão importante são os modelos de informação que produzem as chamadas "Caixas Pretas", processos secretos moldados a partir de dados para informar a pesquisa e a política sob a falsa suposição de representação quando, de fato, as condições em que esses dados foram construídos não são reveladas (Furtado e Renski, 2019). 
Então, o grupo teve o cuidado de buscar as informações e dados da fonte original que era a plataforma Integrasus para ter domínio sobre as informações e não gerar problemas como os citados anteriormente. Todo o processo possibilitou a geração de autonomia por parte dos estudantes envolvidos em relação a manipulação, produção e sistematização de outras bases de dados, gerando conhecimento que também foi compartilhado através da plataforma SOMAR pelos cursos produzidos pela equipe do ArqPET e disponibilizados gratuitamente ${ }^{6}$.

\section{Conclusão}

A experiência do ArqPET UFC na produção de informação da Covid-19 em Fortaleza foi frutífera pois permitiu a visualização e comunicação ampla, assim como proporcionou o estudo espacial da doença para além das leituras trazidas pelos órgãos públicos. Ademais, contribuiu para ampliar o debate sobre dados e tecnologia da informação no grupo, tanto no entendimento dos seus desafios e potencialidades, quanto na sua aplicação para o planejamento urbano e construção de diagnósticos. Esses saberes, principalmente diante de situações emergenciais, são fundamentais para uma atuação efetiva na realidade.

A equipe tinha o intuito de continuar a produzir os dados de acordo com o passar do tempo, até mesmo gerar uma produção para a segunda onda que viera a ocorrer em março de 2021, mas não houve a necessidade visto que a Prefeitura Municipal de Fortaleza passou a disponibilizar os arquivos shapefiles com os dados da covid no site do Fortaleza em Mapas ${ }^{7}$ com incidência por bairro a cada período, o que facilitou bastante o acesso a esse tipo de informação georreferenciada.

\footnotetext{
${ }^{6}$ Acesse em: https://www.somarapet.ora/cursosolidario

${ }^{7}$ Serviço da Prefeitura Municipal de Fortaleza que disponibiliza para o cidadão mapas com informações geográficas produzidas pelos órgãos do município de Fortaleza e secretarias regionais. Acesse em: https://mapas.fortaleza.ce.gov.br/\#l
} 


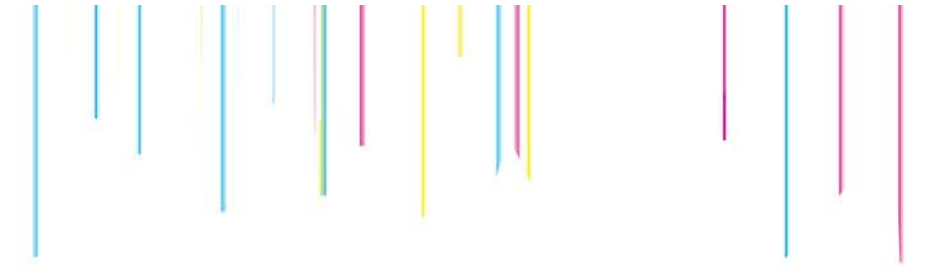

\section{Referências}

Ascher, F. (2009). Os novos princípios do Urbanismo. Romano Guerra Editora.

Arantes, O., VAINER, C., \& Maricato, E. (2000). A cidade do pensamento único: Desmanchando consensos. (3a Edição). Vozes.

Pires, L. N., \& Carvalho, L., Xavier, L. L. (2020). COVID-19 e desigualdade: A distribuição dos fatores de risco no Brasil. Centro Brasileiro de Estudos de Saúde (CEBES). http://dx.doi.org/10.13140/RG.2.2.27014.73282. Acesso dia 21 de julho, 2021, de: http://cebes.org.br/2020/04/covid-19-e-desiqualdade-no-brasil/

ETH Future Cities Laboratory (n. d.) Big Data Informed Urban Design and Governance. Acesso dia 21 de julho,2021, de: https://fcl.ethz.ch/research/fcl-phase2/responsive-cities/biq-data-informed-urbandesia n. html

Furtado, L., \& Renski, H. (2019). Insurgent data building by informal settlements. V!rus, 19. (E-Journal), acesso dia 21 de julho, 2021, de: http://www.nomads.usp.br/virus/ virus19/?sec=4\&item=4\&lanq=en

Harvey, D. (2020). Políticas anticapitalistas em tempos de COVID-19. Jacobin Brasil, acesso dia 21 de julho, 2021, de: https://jacobin.com.br/2020/03/politica-anticapitalista-em-tempos-de-coronavirus/

Observatório das Metrópoles. (2020). Lavar as mãos? Sobre o coronavírus e a precariedade habitacional das nossas cidades. Acesso no dia 21 de julho, 2021, de: https://www.observatoriodasmetropoles.net.br/lavar-as-maos-sobre-

coronavirus-e-a-pr ecariedade-habitacional-das-nossascidades/?utm source=Facebook\&utm medium=Post\&utm campaian=Facebook 627\&utm content=Lavar\%20as\%20m\%C3\%A3os\%3F\%20Sobre\%20coronav\%C3 \%ADrus\%20e\%20a\%20precariedade\%20habitacional\%2 Odas\%20nossas\%20cidades\&fbclid=IwAR1PybAAW9LqT5kBV5LmXoPw6cx0pRhNv zKOKOZzwA3 ih0ivcrH5RBtwc

Tonucci, F., João, B. M., Patrício, P. A., \& Bastos, C. (2020). NOTA TÉCNICA Desafios e Propostas para Enfrentamento da COVID-19 nas Periferias Urbanas: Análise das condições habitacionais e sanitárias dos domicílios urbanos no Brasil e na Região Metropolitana de Belo Horizonte. Centro de Desenvolvimento e Planejamento Regional (Cedeplar) UFMG, acesso dia 21 de julho, 2021, de: https://www.cedeplar.ufmg.br/noticias/1229-nota-tecnica-desafios-e-propostaspara-en\%20\%20\%20frentamento-da-covid-19-nas-periferias-urbanas-analise-dascondicoes-habitacionais-\%20e-sanitarias-dos-domicilios-urbanos-no-brasil-e-naregiao-metropolitana-de-belo-horiz\%20onte 